\title{
Initial results with minimally invasive repair of pectus carinatum
}

\author{
Attila Kálmán, MD
}

Objective: Pectus carinatum is traditionally repaired by using some modification of the open Ravitch procedure, with its possible long-term sequelae, such as poor postoperative compliance of the chest. In this study we assessed our results with a new minimally invasive repair of pectus carinatum that requires neither cartilage incision nor sternotomy.

\begin{abstract}
Methods: From June 2005, we have corrected pectus carinatum using a method analogous to the Nuss procedure for pectus excavatum repair. Thus far, we performed this intervention on 14 patients (mean age, $15 \pm 1.5$ years). A steel bar has been inserted at the level of the maximum point of the sternal protrusion through small lateral incisions. The sternum is pushed back without osteotomy or chondrotomy. The bar is removed after 2 years. Patients' characteristics, operation time, hospital stay, and complications have been recorded.
\end{abstract}

Results: In 1 patient with asymmetric deformity, 2 bars were placed. Operative time was $42 \pm 20$ minutes (mean \pm standard deviation), and hospital stay was 3 days (median quartiles, 3-4 days) postoperatively. We experienced lateral shift of the bar in 1 patient, which was treated with remodeling and repositioning of the bar. No other complication occurred during the 18-month follow-up period (mean range, 2-38 months). Thirteen of the 14 patients reported excellent or very good results. Patients returned to full activity within 2 months. Five bars have been removed.

Conclusions: Minimally invasive repair of pectus carinatum leaves the integrity of the chest wall untouched. It is safe with a short operative time and hospital stay and provides good results, even in asymmetric cases.

With the worldwide spread of the revolutionary new minimally invasive repair of pectus excavatum (MIRPE) or Nuss procedure, ${ }^{1}$ strong demand has emerged for minimally invasive repair of pectus carinatum (PC).

Abramson ${ }^{2}$ has described a method applying the principle of the Nuss procedure: use of a bar for the correction of the chest deformity by compressing the protruding sternum in its desired position and maintaining the correction long enough for permanent remolding of the chest. He places the bar anteriorly to the sternum and fixes it to the ribs with wires posteriorly. He has reported excellent results. Because the bar is totally outside the chest wall, the whole force against the sternum is sustained by the wires.

Accepting the principle of the Nuss procedure, we decided to thread the chest wall on a properly modeled bar in the corrected position, providing greater stability and usability in severe and asymmetric cases. The middle part of the bar is anterior to the sternum. It is driven into the chest on both sides parasternally, and both ends are brought out to the outer surface of the ribs. In this way the bar exerts maximal force to constrain the chest wall in the desired position. Because the bar goes through the chest wall 4 times, it can-

From the First Department of Pediatrics, Semmelweis University, Budapest, Hungary. Received for publication Sept 6, 2008; revisions received Oct 26, 2008; accepted for publication Dec 25, 2008; available ahead of print March 9, 2009.

Address for reprints: Attila Kálmán, MD, 1st Department of Pediatrics, Semmelweis

University, Bókay u. 53, 1083 Budapest, Hungary (E-mail: ka@gyer1.sote.hu).

J Thorac Cardiovasc Surg 2009;138:434-8

$0022-5223 / \$ 36.00$

Copyright (c) 2009 by The American Association for Thoracic Surgery doi: $10.1016 /$ j.jtcvs.2008.12.032 not rotate, and it is able to correct asymmetric deformities also if the chest is not extremely rigid.

This report presents the surgical technique and results obtained with this minimally invasive procedure in 14 patients.

\section{MATERIALS AND METHODS}

From June 2005 to June 2008, 14 consecutive patients with the chondrogladiolar form of PC underwent operations at the author's institute using his technique; all the operations were performed by the author. All patients with PC repair were prospectively followed up. Age of the patients was $15 \pm 1.5$ years (mean \pm standard deviation). The deformity developed after 10 years of age in 7 of the 14 patients ( 13 boys and 1 girl). Two patients experienced mild exercise intolerance before the operation. In 4 boys the deformity was markedly asymmetric, with a protruding parasternal region on 1 side.

The operation is performed after achievement of general endotracheal anesthesia with muscle relaxation. In most cases epidural analgesia is used for pain control in the postoperative period. All patients are given a 2-day course of amoxicillin-clavulanic acid starting at the time of the operation.

The patient's chest is measured before surgical intervention, and the appropriately sized bar is bent. A T-bar with a fixed transverse part at one end of the bar (Kálmán T-bar; Sanatmetal Ltd, Eger, Hungary) is used. It is made of surgical steel and is $15 \mathrm{~mm}$ wide and 2 or $3 \mathrm{~mm}$ thick, depending on the bar's length. The bar reaches the midaxillary line on both sides and is modeled with multiple curves (Figure 1).

Small 4-cm-long incisions are made on both sides in the midaxillary line at the level of the maximum point of sternal protrusion while the patient is in the supine position with both arms abducted. The thoracic cavity is entered with a long curved Kelly clamp on both sides. Starting from these points, the inner surface of the thoracic wall is checked for any adhesions in medial direction to the lateral border of the sternum by using the index fingers. Keeping the tip of the instrument against the inner surface of the chest wall, it is directed medially in a horizontal direction. It is led out from the chest parasternally through the next intercostal space, and a presternal subcutaneous tunnel is created over the protrusion by working from both sides (Figure 2, A). During this phase of the operation, special care is taken of the skin. As 


\section{Abbreviations and Acronyms \\ MIRPE $=$ minimally invasive repair of pectus excavatum \\ $\mathrm{PC} \quad=$ pectus carinatum}

the track of the bar is created in its full length, the bar is introduced from the right side with its concavity facing anteriorly. As the tip of the bar reaches the presternal tunnel, it is advanced while it is rotated (Figure 2, B). The tip of the bar is driven into the chest on the left side parasternally, keeping it against the index finger of the surgeon (Figure 2,C). As the bar reaches the hole on the lateral chest wall on the left side, its end is pulled out to the outer surface of the ribs (Figure 2,D). The bar is fixed to the lateral chest wall muscles on both sides with absorbable sutures. Air is aspirated from the chest, and the wounds are closed without leaving drains. A chest radiograph is obtained in the operating room.

Approval by the institutional review board and informed consent from each participant were obtained.

The postoperative appearance of the chest was judged by the patients and their parents as excellent, very good, good, fair, or poor.

\section{RESULTS}

In 1 patient with marked asymmetry, a second bar was placed in an asymmetric position under the left costal arch. Blood loss was minimal (up to approximately $10 \mathrm{~mL}$ ). No patient required transfusion. Operative time was $42 \pm 20$ minutes (mean \pm standard deviation). Our patients required epidural catheter or narcotic pain medication for $34 \pm 14$ hours. Hospital stay was 3 days (median quartiles, 3-4 days) postoperatively. We experienced lateral shift of the bar in our oldest (18.5 years old) patient because of imprecise modeling and placement of the bar. This was treated with remodeling and repositioning of the bar. We had no other complication during the follow-up period of 18 months (mean range, 2-38 months). All deformities, even the asymmetric cases, were corrected (Figures 3-5). Thirteen of the 14 patients experienced excellent or very good results, and 1 patient reported a good result. All patients returned to full activity within 2 months.

We removed the bar from our first 5 patients $25 \pm 3$ months after the correction of their deformities. We could remove the bar without any difficulty in $12 \pm 3$ minutes, and all the patients were discharged on the day after removal of the bar. The corrected shape of their chests remained stable during the follow-up period of 2 to 14 months since bar removal.

\section{DISCUSSION}

$\mathrm{PC}$ is characterized by the protrusion of the sternum resulting from overgrowth of the costal cartilages. It has different subtypes depending on the maximum point of the deformity. The chondrogladiolar form is the most common variety, with symmetric or asymmetric protrusion of the gladiolus and inferior costal cartilage. The manubrium and the superior costal cartilages protrude in the chondromanub-

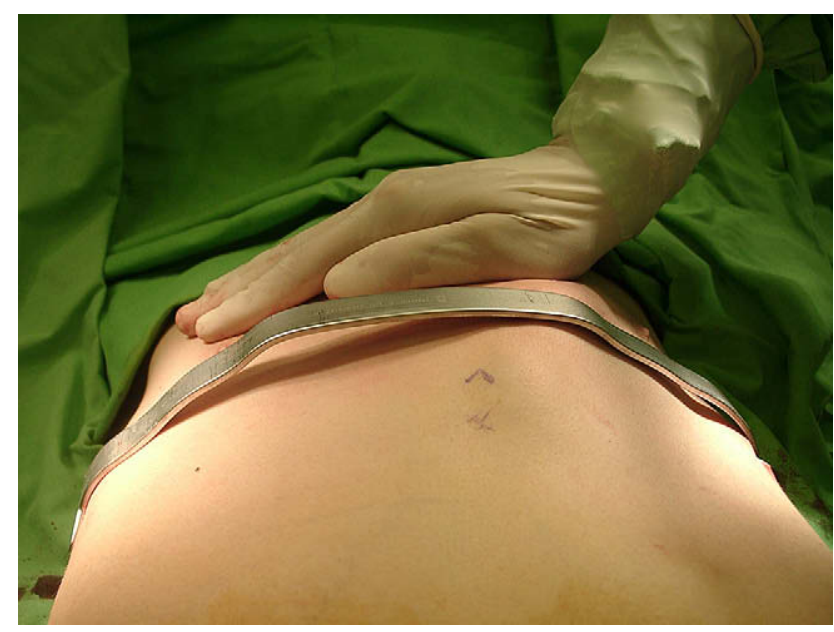

FIGURE 1. The molded bar before insertion.

rial form, which is much less common. In almost one half of the affected children, the deformity is not appreciated until after the 10th birthday, and it usually worsens during the active linear growth of puberty. The condition occurs more frequently in boys than girls (4:1). Recommendation for surgical correction remains controversial because PC is rarely associated with significant cardiopulmonary derangements. However, it can have severe psychological effects in adolescents.

Usually, the Ravitch procedure or its modified versions are used for the repair of PC. ${ }^{3-6}$ The key steps of these procedures are similar to those of the Ravitch procedure used for pectus excavatum repair: anterior chest wall incision, elevation of the pectoralis major muscles, subperichondrial resection of the deformed costal cartilages bilaterally, transverse sternotomy, and fixation of the corrected shape of the chest wall. Although these provide good results, they are major operations with long operating times, significant blood loss, and, possibly, long-term consequences, such as upper thoracic overgrowth or poor postoperative compliance of the chest. A reduced level of performance on pulmonary function tests was documented because of scarring and rigidity of the anterior thoracic wall after thoracoplasty for pectus excavatum deformities. ${ }^{6-9}$ Furthermore, if thoracoplasty is performed too early in childhood, asphyxiating chondrodystrophy might develop because of the risk of interference with growth plates. ${ }^{10,11}$ There are no similar studies on long-term problems regarding traditional PC repair, probably because of the lower number of patients undergoing the Ravitch procedure for PC. However, based on the similarity of the main steps, the long-term effect of the Ravitch procedure on chest wall mechanics must be the same for patients with PC as for those with pectus excavatum.

Mainly for these reasons, only severe cases of PC are repaired, and the procedure should be reserved for patients who have completed their growth. 

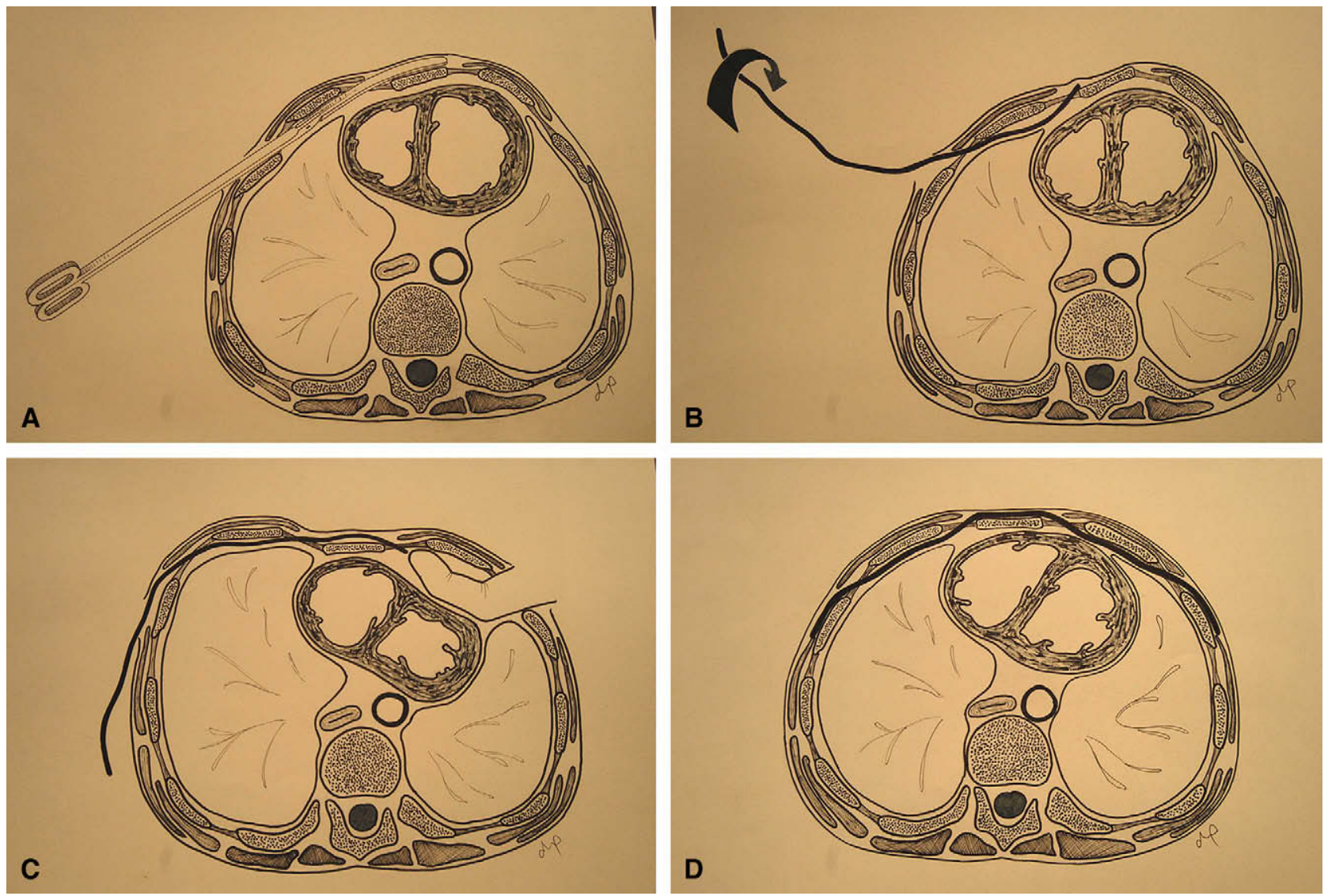

FIGURE 2. A, A presternal subcutaneous tunnel is created over the protrusion by working from both sides. B, The bar is introduced from the right side. The bar is advanced in the presternal tunnel while it is rotated. C, The bar is driven into the chest, keeping it against the index finger. D, Final position of the bar.

A compressive orthotic brace has been developed for nonoperative treatment of PC. The pliability of the chest wall seen in most of these patients makes it a reasonable method, especially in the chondrogladiolar form of the deformity. Frey and colleagues ${ }^{12}$ and Egan and associates ${ }^{13}$ reported good re- sults, but wearing the brace for 14 to 16 hours a day necessitates long-term strong compliance of the patients. We did not realize similar good results in our few patients with orthotic braces.

After May 2000, when we performed our first MIRPE, many patients had been referred to our department for

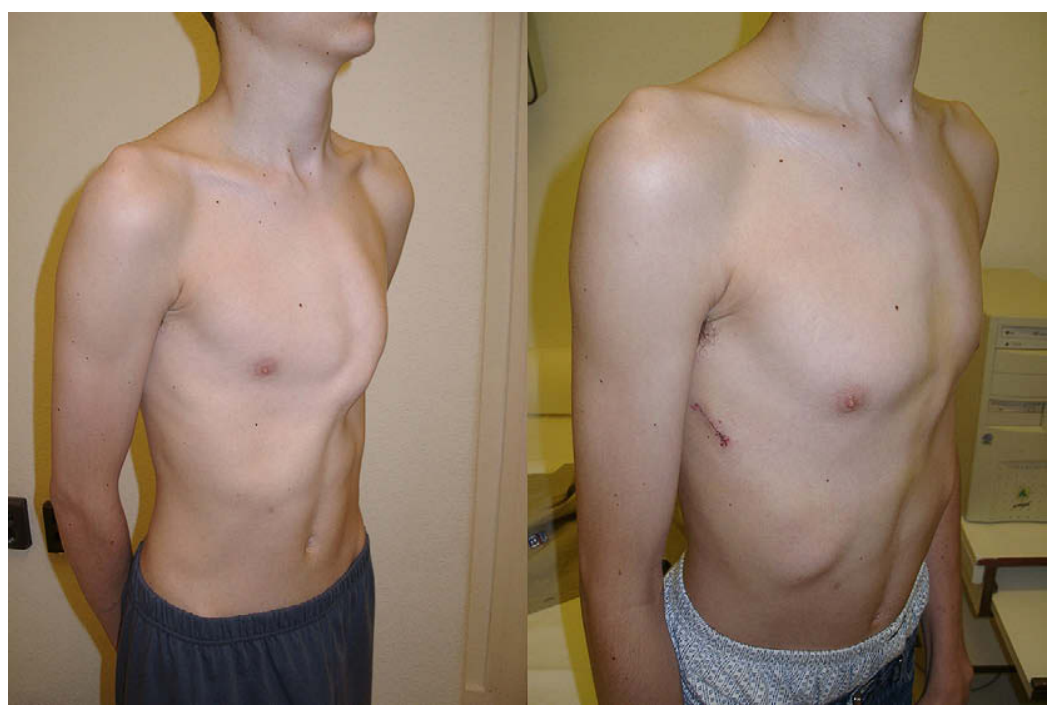

FIGURE 3. Asymmetric pectus carinatum before and after the intervention (right lateral view). 


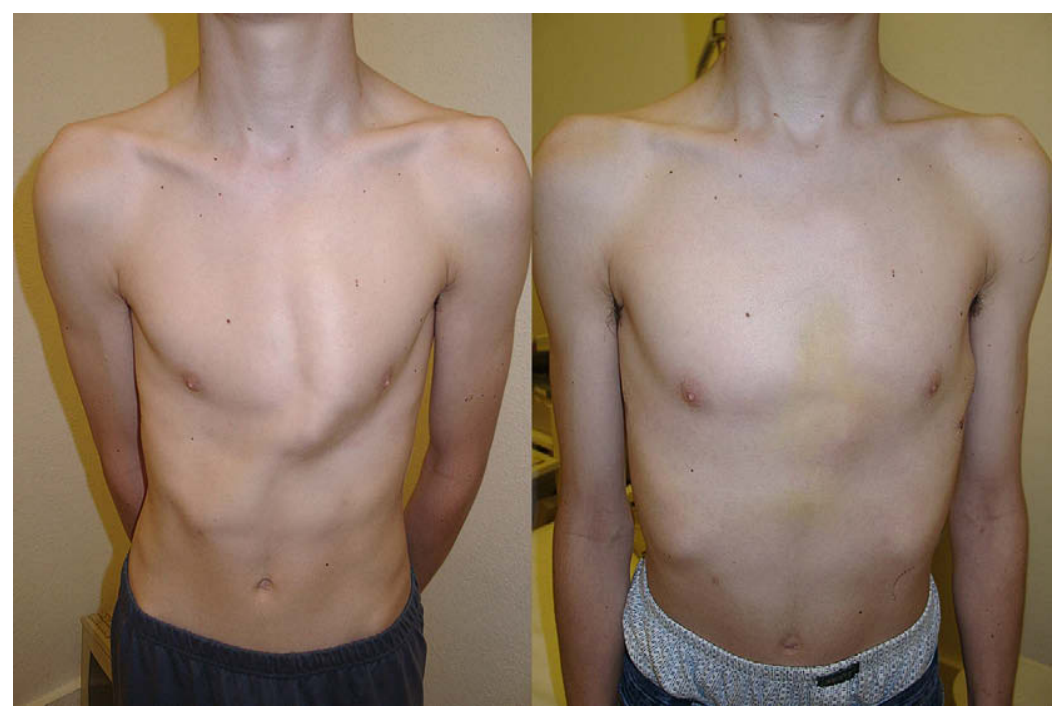

FIGURE 4. Asymmetric pectus carinatum before and after the intervention (frontal view).

evaluation of their chest wall deformity. For the patients with pectus excavatum deformity who were judged to warrant reconstructive surgery, we could offer minimally invasive repair, although not for the patients with PC deformity.

With the experience of more than 200 MIRPEs, we started to perform minimally invasive correction of PC in June 2005. Abramson's method ${ }^{2}$ was the only report on minimally invasive repair of PC published in the literature. For theoretical reasons (ie, increased stability and suitability in pronounced or asymmetric cases), we decided to use the steel bar in a different way. The chest wall is threaded on the modeled bar, and it is constrained into the desired position. By using manual control during insertion of the bar, checking possible adhesions, and driving the bar into the thoracic cavity, major complications, such as cardiac or lung injury, can be prevented.

All but 1 of our patients were between 13 and 16.5 years of age. Their chests were fairly malleable, permitting correction of the deformity with this minimally invasive method. The pliability of the chest wall in these patients might contribute to the lesser requirement for analgesia than that seen in patients undergoing operations for pectus excavatum. In our oldest patient (18.5 years old), less pliability of her chest might have contributed to the lateral shift of the bar. No strict age but rather the rigidity of the chest wall can limit the use of our minimally invasive repair.

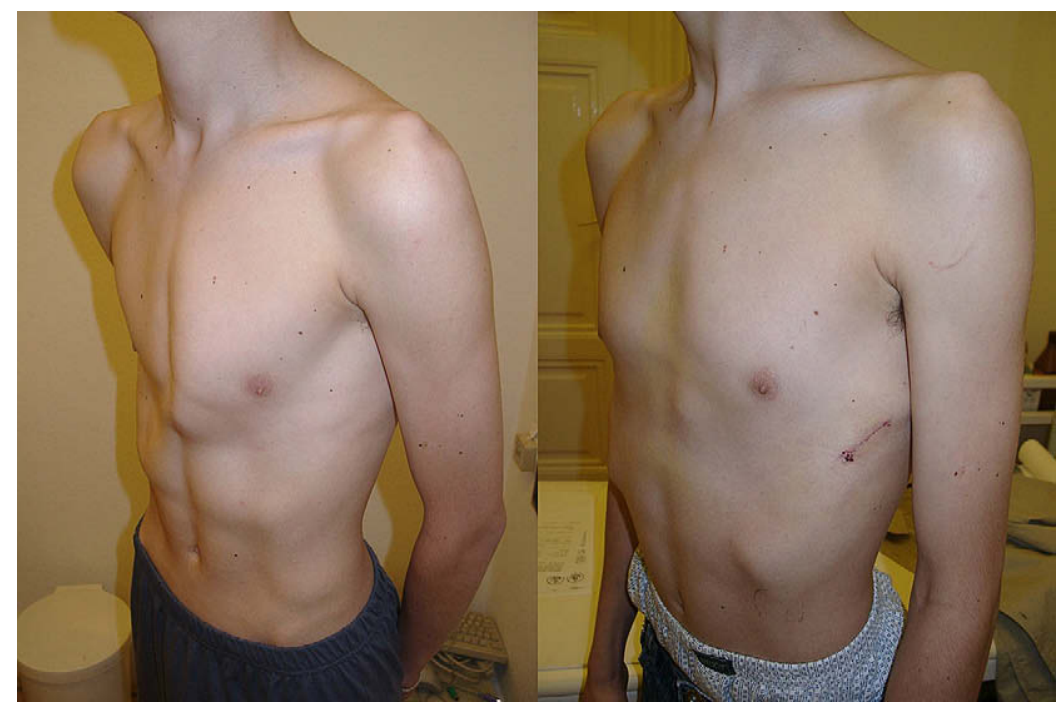

FIGURE 5. Asymmetric pectus carinatum before and after the intervention (left lateral view). 
Although we have rather promising experience in cases of asymmetric cases as well, in severely asymmetric cases traditional open procedure is an option other than the presented minimally invasive procedure. In these cases we always have to weigh the cost and benefit of each procedure. The patient has to choose between the less invasive technique with possible residual deformity and the traditional Ravitch operation, which is much more invasive and leaves an obvious scar but occasionally can offer better shape of the thoracic wall.

By using experience learned from pectus excavatum and its minimally invasive repair, the bar is left in place as a splint for 2 years.

\section{CONCLUSIONS}

Our limited experience suggests that minimally invasive repair of PC is feasible, and after more experience is gained, it can become a real alternative to traditional thoracoplasty in the chondrogladiolar form of PC. The advantages of this procedure are (1) no anterior chest wall incision, with the integrity of the chest wall remaining untouched; (2) short operating time and minimal blood loss; (3) short hospital stay and early return to activity; and (4) safety with excellent results. The properly modeled bar (passing the thoracic wall 4 times) might be able to correct asymmetric deformities also by forcing the chest wall to take the desired shape. Further experience with more patients is necessary to evaluate the long-term results and to study whether minimally invasive repair is applicable in the chondromanubrial form of PC.

\section{References}

1. Nuss D, Kelly RE, Croitoru DP, Katz ME. A 10-year review of a minimally invasive technique for correction of pectus excavatum. J Pediatr Surg. 1998;33: 545-52.

2. Abramson H. A minimally invasive technique to repair pectus carinatum. Preliminary report. Arch Bronconeumol. 2005;41:349-51.

3. Ravitch MM. The operative correction of pectus carinatum (pigeon breast). Ann Surg. 1960;151:705-14

4. Welch KJ, Vos A. Surgical correction of pectus carinatum (pigeon breast). JPediatr Surg. 1973;8:659-67.

5. Fonkalsrud EW, Beanes S. Surgical management of pectus carinatum: 30 years' experience. World J Surg. 2001;25:898-903.

6. Shamberger RC. Congenital chest wall deformities. Curr Probl Surg. 1996;33: 469-552.

7. Morshuis W, Folgering H, Barentsz J, van Lier H, Lacquet L. Pulmonary function before surgery for pectus excavatum and at long-term follow-up. Chest. 1994; 105:1646-52.

8. Lacquet LK, Morshuis WJ, Folgering HT. Long-term results after correction of anterior chest wall deformities. J Cardiovasc Surg. 1998;39:683-8.

9. Derveaux L, Ivanoff I, Rochette F, Demedts M. Mechanism of pulmonary function changes after surgical correction for funnel chest. Eur Respir J. 1988;1:823-5.

10. Martinez D, Juame J, Stein T, Pena A. The effect of costal cartilage resection on chest wall development. Pediatr Surg Int. 1990;5:170-3.

11. Haller JA, Colombani PM, Humphries CT, Azizkhan RG, Loughlin GM. Chest wall constriction after too extensive and too early operations for pectus excavatum. Ann Thorac Surg. 1996;61:1618-25.

12. Frey AS, Garcia VF, Brown RL, Inge TH, Ryckman FC, Cohen AP, et al. Nonoperative management of pectus carinatum. J Pediatr Surg. 2006;41:40-5.

13. Egan JC, DuBois JJ, Morphy M, Samples TL, Lindell B. Compressive orthotics in the treatment of asymmetric pectus carinatum-a preliminary report with an objective radiographic marker. J Pediatr Surg. 2000;35:1183-6. 